\section{Neue Technologien und Monitoringmethoden in der Fließgewässerforschung}

Die Herausforderungen, den europäischen Umweltzielen für unsere Fließgewässer gerecht zu werden, sind aufgrund der vielfachen unterschiedlichen Nutzungen kaum integrativ fassbar bzw. aufgrund derzeitiger technischer Möglichkeiten teilweise nur eingeschränkt implementierbar. Um letztendlich ein passendes Management zu erzielen, braucht es detaillierte und weiterführende Untersuchungsmethoden - in Ergänzung zu der gemäß EU-Wasserrahmenrichtlinie vorgegebenen nationalen Überwachung. In diesem Sonderheft werden neue und innovative Konzepte zum Umweltmonitoring mit dem Schwerpunkt auf Fließgewässer vorgestellt, bzw. neue technische Möglichkeiten beschrieben, um negative Auswirkungen auf die Gewässerökologie zu vermindern.

Im Fachbeitrag von Flödl et al. wurde ein neuer stationärer Organik-/Anorganik-Sammler entwickelt. Hintergrund ist die Notwendigkeit, die anthropogen verursachte Schadstoffbelastung, welche für Ökosysteme ein potenzielles Risiko darstellt, besser zu erfassen. Die hohe Anzahl an Schadstoffen, die oft auch nur in sehr geringen Konzentrationen in der aquatischen Umwelt auftreten, erschwert jedoch deren Nachweis. Die Erforschung der Eintragspfade in Fließgewässer, die Schadstoffmobilität sowie deren mögliche Folgen stellen daher eine besondere Herausforderung dar. Für die Ermittlung der Schadstoffkonzentrationen in kleinen Gewässern mit geringen Fließgeschwindigkeiten gibt es nur eine kleine Auswahl an Messmethoden, die jedoch meist eine gute Infrastruktur vor Ort benötigen. In dem Beitrag von Flödl et al. wird gezeigt, dass eine adaptierte Version eines mobilen Geschiebefängers zum Sammeln einer ausreichenden Menge an Feinmaterial (Korngrößen $<1 \mathrm{~mm}$ ) sowie an Schwimmstoffen genutzt werden kann, um diese anschließend im Labor auf Schadstoffkonzentrationen untersuchen zu können. Die ersten Untersuchungen zeigen hierbei interessante Erkenntnisse in Bezug auf die Verteilung der Schadstoffkonzentrationen und auf das Forschungspotenzial im Bereich des Schadstoffeintrags und -transports in kleinen Einzugsgebieten $\left(<100 \mathrm{~km}^{2}\right)$.

In der Publikation von Mader et al. wird ein neues fischökologisches Monitoring-System 4.0 mittels FishCam und FishNet vorgestellt. Dieses System hat zum Ziel, automatisierbare Aufgaben, wie die präzise, ausdauernde und rund um die Uhr einsetzbare Erfassung von Felddaten und die Vorauswertung derselben, mit den finalen Expertisen einer Artbestimmung und Bewertung durch Fachkräfte zu verknüpfen. Mit dem neu entwickelten System zur Felddatenerfassung mittels LAN-Kamera in einem Reinwassergehäuse und einem Erfassungstunnel wird die Fischmigration automatisch ohne hydraulischen Einfluss, ohne Hälterung der Fische in Reusen und ohne Kontakt und Stress für die Fische erfasst. Die angewandte Bildklassifizierung in Fish- und NoFish-Objekte erfolgt auf Basis eines trainierten Deep Convolutional Neural Network und erreicht eine Genauigkeit in der Differenzierung von ca. $97 \%$. Basierend auf der sekundengenauen Erfassung der Wanderzeiten der Einzelindividuen erfolgten über Sonderauswertungen die Analyse eines Blockadeeffekts bei den Annäherungen der Fische zu intermittierend arbeitenden Organismenwanderanlagen sowie die Analyse der Auffindbarkeit, der Tages- und Jahresgänge der Fischwanderung und die Auswertung von Massenwanderungen.

In einem weiteren Fachbeitrag von Pulg et al. wird für Mitteleuropa ein möglicher neuer Stressor für alpine Fließgewässer beschrieben. Die sogenannte Gasblasenkrankheit bei aquatischen Organismen ist in Europa nur wenig beachtet. Neue Forschungsergebnisse in Norwegen, China und Nordamerika zeigen jedoch, dass Gasübersättigung verursacht durch Wasserkraftwerke weitaus stärker verbreitet ist als bisher angenommen. In Norwegen wurde beispielsweise eine künstliche Gasübersättigung in 12 von 16 untersuchten Wasserkraftwerken nachgewiesen. Dabei kam es in 10 Fällen zu Fischsterben.

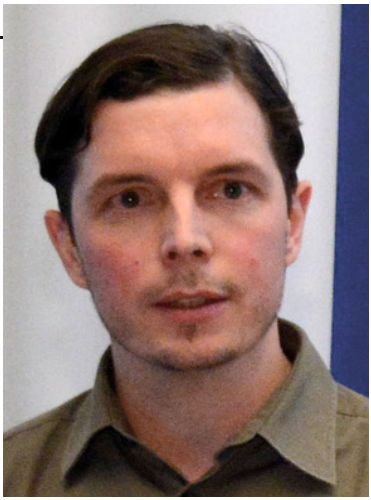

PD DI Dr. Christoph Hauer

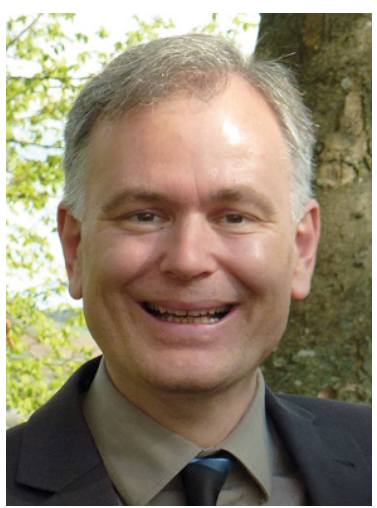

Univ.-Prof. DI Dr. Helmut Habersack 


\section{Editorial}

Ursache ist der Eintrag von Luft in die Zuleitungen, welche sich unter Druck im Wasser löst. In vielen Fällen kann die Gasübersättigung vermieden werden, beispielsweise mit ausreichender Dimensionierung von Kraftwerkseinläufen und Rechenanlagen, Alarmsystemen und Deflektoren. Umwelteffekte und die Toleranz heimischer Arten sind in Europa jedoch kaum erforscht. Das Gleiche gilt für die Verbreitung künstlicher Gasübersättigung in Europa sowie für kosteneffektive Methoden zu ihrer Vermeidung an bestehenden Anlagen. Im Fachartikel Pulg et al. werden verschiedene Aspekte der Gasübersättigung thematisiert. Neben einer Beschreibung der physikalischen Prozesse werden sowohl die biologischen Auswirkungen als auch Messmethoden und mögliche Maßnahmen präsentiert.

Möglichkeiten zur innovativen Steuerung von Kleinwasserkraftwerken werden in Reiter et al. beschrieben. Anthropogene Wasserspiegelschwankungen besitzen wesentliche negative Auswirkungen auf die aquatische Fauna und Flora. Diese als Sunk/Schwall bezeichneten Wasserspiegeländerungen wurden bisher hauptsächlich in Bezug auf die Großwasserkraft untersucht und mögliche Verbesserungsmaßnahmen wurden für diese speziellen Wasserkrafttypen erarbeitet. Auch im Bereich der Kleinwasserkraft besteht durch betriebstechnische Probleme bzw. auch Stör- und Unfälle die Möglichkeit, dass kurzfristige Abfluss- und Wasserspiegelschwankungen entstehen, die sich aufgrund von automatisierten Steuerungen in einem Fließgewässer über eine Kraftwerkskette hinweg weiter fortsetzen. Im Fachbeitrag von Reiter et al. werden, neben einer Darstellung der allgemeinen Problematik, vor allem neue Methoden zur Steuerung von Kleinwasserkraftanlagen vorgestellt, die mitunter die einzige Möglichkeit für eine gezielte Verbesserung bieten.

Der abschließende Fachbeitrag in diesem Sonderheft von Schenekar et al. beinhaltet eine Vorstellung der Möglichkeiten, ein Fließgewässer mittels eDNA zu monitoren. In den letzten Jahren wurden vermehrt DNA-basierte Methoden zur Unterstützung konventioneller Ansätze von Biodiversitätserhebungen vorgeschlagen. Hierbei wird der Verwendung von Umwelt-DNA (oder environmental DNA eDNA) ein besonders großes Potenzial zur Verbesserung von Biomonitorings zugeschrieben. Anhand von eDNA können mittels Einzelartansätzen wie qPCRs gezielt Arten nachgewiesen, oder, mittels Metabarcoding, die gesamte Artenzusammensetzung einer Zielgruppe beschrieben werden. In dem Artikel von Schenekar et al. wird ein kurzer Überblick über Potenzial und Rahmenbedingungen dieser Methoden gegeben und es werden drei kleine Pilotstudien von eDNA-Analysen in österreichischen Fließgewässern vorgestellt. Des Weiteren werden die Vor- und Nachteile von eDNA basierten Ansätzen im Vergleich mit konventionellen Methoden diskutiert.

PD DI Dr. C. Hauer ( $₫)$ · Univ.-

\section{Prof. DI Dr. H. Habersack}

Christian Doppler Labor für

Sedimentforschung und -management, Institut für Wasserbau, Hydraulik und Fließgewässerforschung, Department für Wasser-Atmosphäre-Umwelt, Universität für Bodenkultur Wien, Muthgasse 107,

1190 Wien, Österreich

christoph.hauer@boku.ac.at

Univ.-Prof. DI Dr. H. Habersack

helmut.habersack@boku.ac.at 